\title{
Bioisosteric modification on melatonin: synthesis of new naphthalene derivatives, in vitro antioxidant activity and cytotoxicity studies
}

\author{
Hanif Shirinzadeh ${ }^{\circledR 1^{*}}$, Mohammed Ghalia², Alev Tascioglu ${ }^{3}$ Ferial Intissar Adjali², \\ Gulsen Gunesacar ${ }^{1}$, Hande Gurer-Orhan ${ }^{3}$, Sibel Suzen $^{4}$
}

\begin{abstract}
${ }^{1}$ Department of Pharmaceutical Chemistry, Faculty of Pharmacy, Erzincan Binali Yildirim University, Yalnizbag, Erzincan, Turkey, ${ }^{2}$ Department of Chemistry, Université Mentouri Constantine, Constantine, Algérie, ${ }^{3}$ Department of Pharmaceutical Toxicology, Faculty of Pharmacy, Ege University, Izmir, Turkey, ${ }^{4}$ Department of Pharmaceutical Chemistry, Ankara University, Faculty of Pharmacy, Ankara, Turkey
\end{abstract}

\begin{abstract}
Melatonin (MLT) is a strong free radical scavenger that protects the body from the deleterious effects of excess oxidants. Synthesis of MLT analogue compounds with antioxidant potency has recently attracted the interest of researchers. In general, the strategy consists of modifying the groups in the different sites of the indole ring or replacing the indole ring with an analogue. As part of our ongoing research, the antioxidant capacity and cytotoxicity of newly synthesized MLT analogue naphthalene derivatives were evaluated. The radical scavenging activity was tested by a 1,1-diphenyl-2-picrylhydrazyl (DPPH) assay. Most of the synthesized compounds showed significant antioxidant activity in comparison to MLT. The structure-activity relationship was identified. The in vitro cytotoxic effects of the synthesized compounds were also investigated in CHO-K1 cells using the MTT assay.
\end{abstract}

Keywords: Antioxidant. Cytotoxic. Melatonin. MTT. DPPH.

\section{INTRODUCTION}

Melatonin (MLT) is a neurohormone secreted by the pineal gland in the human body (Maharaj et al., 2005). Biosynthesis of MLT ( $\mathrm{N}$-acetyl-5-methoxytrypamine) starts with the amino acid L-tryptophan which is a precursor amino acid for synthesizing both serotonin and MLT in the pineal gland (Yonei et al., 2010). MLT has been recognized as a specific hormone of the pineal gland, but it is also secreted in the gastrointestinal tract, skin, retina, brain and some other parts of the body (Reiter et al., 2000; Suzuki et al., 2008). MLT and its derivatives are responsible of the regulation of the circadian rhythm. In addition to this important function, MLT performs numerous tasks in the body such as oncostatic effects, immune modulation and antioxidant activities (Danilov, Kurganova, 2016). Overproduction of reactive oxygen species (ROS) can cause oxidative stress (OS), which may

\footnotetext{
*Correspondence: H. Shirinzadeh. Department of Pharmaceutical Chemistry, Faculty of Pharmacy, Erzincan Binali Yildirim University, 24100, Yalnizbag, Erzincan, Turkey. Tel: +90 446 2245344; Fax: +90 446 2245343. E-mail: hanif.shirinzade@gmail.com
}

lead to vital damage to cell structures such as proteins and DNA(Valko et al., 2007; Yamashita et al., 2013). MLT and its metabolites have noteworthy antioxidant properties and are able to function as endogenous free-radical scavengers (Reiter et al., 1999; Shirinzadeh et al., 2016). According to recent research, MLT has protective effects in some of OSrelated diseases such as Huntington's disease, Parkinson's disease, Alzheimer's disease and ageing (Suzen, 2007; Allegra et al., 2003; Suzen et al., 2013; Carocci, Catalano, Sinicropi, 2014).

Long-term studies on the MLT molecule brought about only a few commercialized MLT analogues such as Circadin, ramelteon, agomelatine and Tasimelteon (Lemoine, Zisapel, 2012; Hirai et al., 2005). Substitution of the indole ring of MLT with other rings which are isosteres for indole was of major interest. One of the isostere rings is naphthalene (Landagaray et al., 2016). Agomelatine is the first naphthalenic analogue of MLT that was confirmed as an antidepressant agent for treatment of major depressive disorders by the European Medicines Agency (Ettaoussi et al., 2012). Many studies have reported the antioxidant properties of 
agomelatine (Aguiar et al., 2013). As part of our ongoing research, naphthalene derivatives analogous to MLT were synthesized (De Mello et al., 2016; Azim, Agarwal, Vohora, 2017). In general, the strategy in this study consisted of replacing the indole ring with the bioisosteric naphthalene ring. All twenty-one compounds except $\mathbf{1 h}$ (Robev, 1981), 1r (Robev, 1968) and 1u (Weil, Ostermeier, 1921; Ding et al., 2017) were new. Furthermore, the compounds $\mathbf{1 n}, \mathbf{1 p}$ and 10 have CAS registry numbers, but there is no information in the literature related to the biological activity of these. The synthesized compounds were characterized on the basis of ${ }^{1} \mathrm{H}$ and ${ }^{13} \mathrm{C}$ NMR, mass spectra and elemental analyses. The biological activity of the compounds was investigated in in vitro conditions by a well-known 1,1-diphenyl 2-picrylhydrazyl (DPPH) radical scavenging activity assay. The in vitro cytotoxic effects of the synthesized compounds were investigated in CHO-K1 cells using the MTT assay.

\section{MATERIAL AND METHODS}

This study was designed to synthesize, characterize and investigate the potential antioxidant and cytotoxic effects of new MLT analogue compounds containing the naphthalene ring instead of the indole ring. Two major modifications may be seen on MLT. The first one was the replacement of the indole ring of MLT with naphthalene which is bioisosteric with indole. The second modification was made on the acetylamino-ethyl side chain by formation of imine (Figure 1). Two series of new compounds were synthesized (Table I). It is known that there are different opinions about the antioxidant efficacy of the methoxy group in the MLT molecule (Shirinzadeh et al., 2010; Spadoni et al., 2006; Letra-Vilela et al., 2016). To identify the antioxidant role of the methoxy group on the naphthalene ring, 6-methoxy naphthalene and nonmethoxy naphthalene series were synthesized.

\section{Chemistry - experimental}

Uncorrected melting points were determined with a Stuart melting point SMP30 apparatus. The ${ }^{1} \mathrm{H}$ and ${ }^{13} \mathrm{C}$ NMR spectra were measured with a Varian 400 $\mathrm{MHz}$ spectrometer device (Palo Alto, CA) using TMS as an internal standard and DMSO- $\mathrm{d}_{6}$ as solvent. ESI mass spectra were determined by a Waters Micromass ZQ device. Elemental analyses were performed using a CHNS-932 instrument (Leco Corporation, St. Joseph,

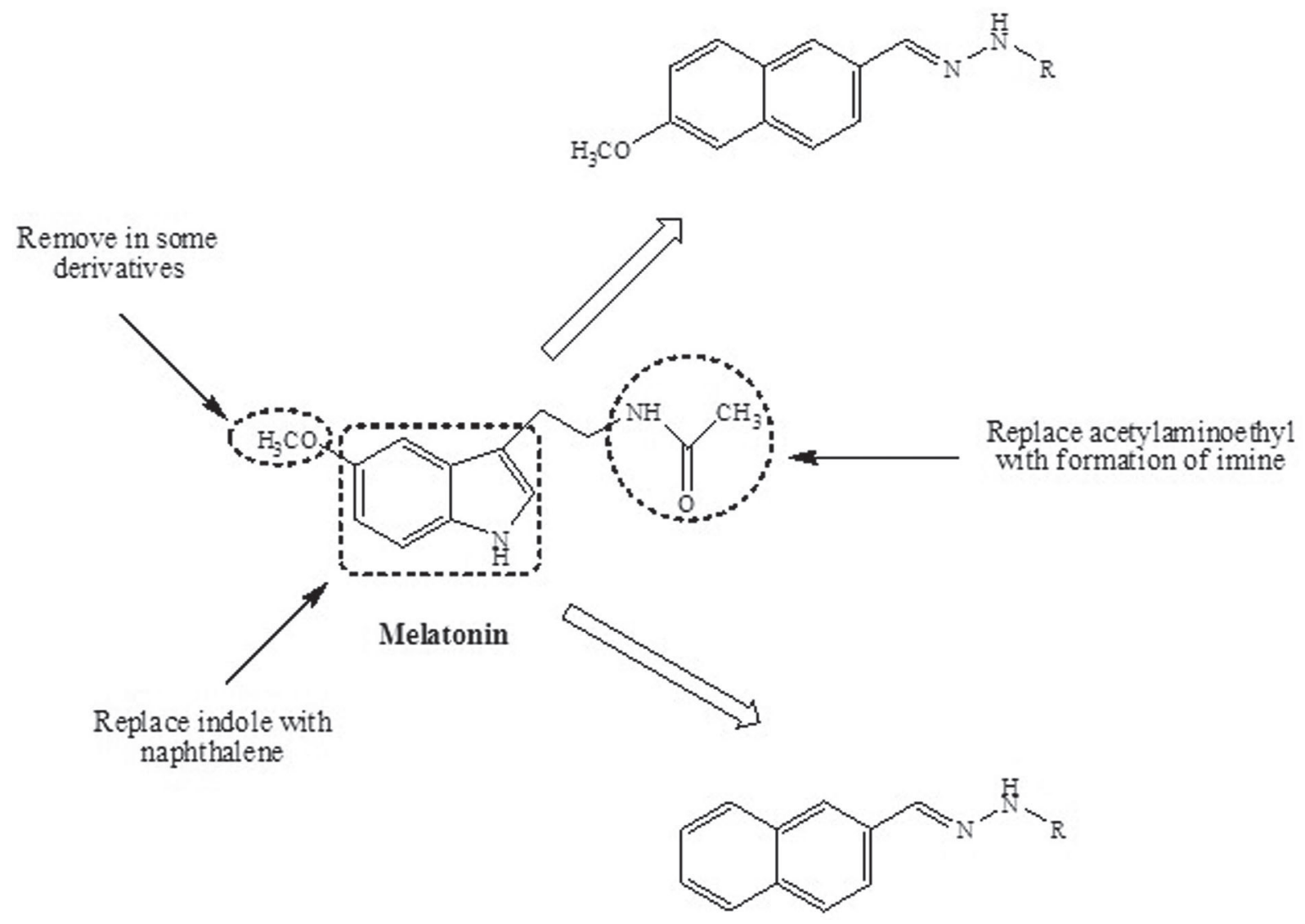

FIGURE 1 - Modifications on MLT molecule to develop new analogues. 
TABLE I - Chemical structures of newly synthesized compounds

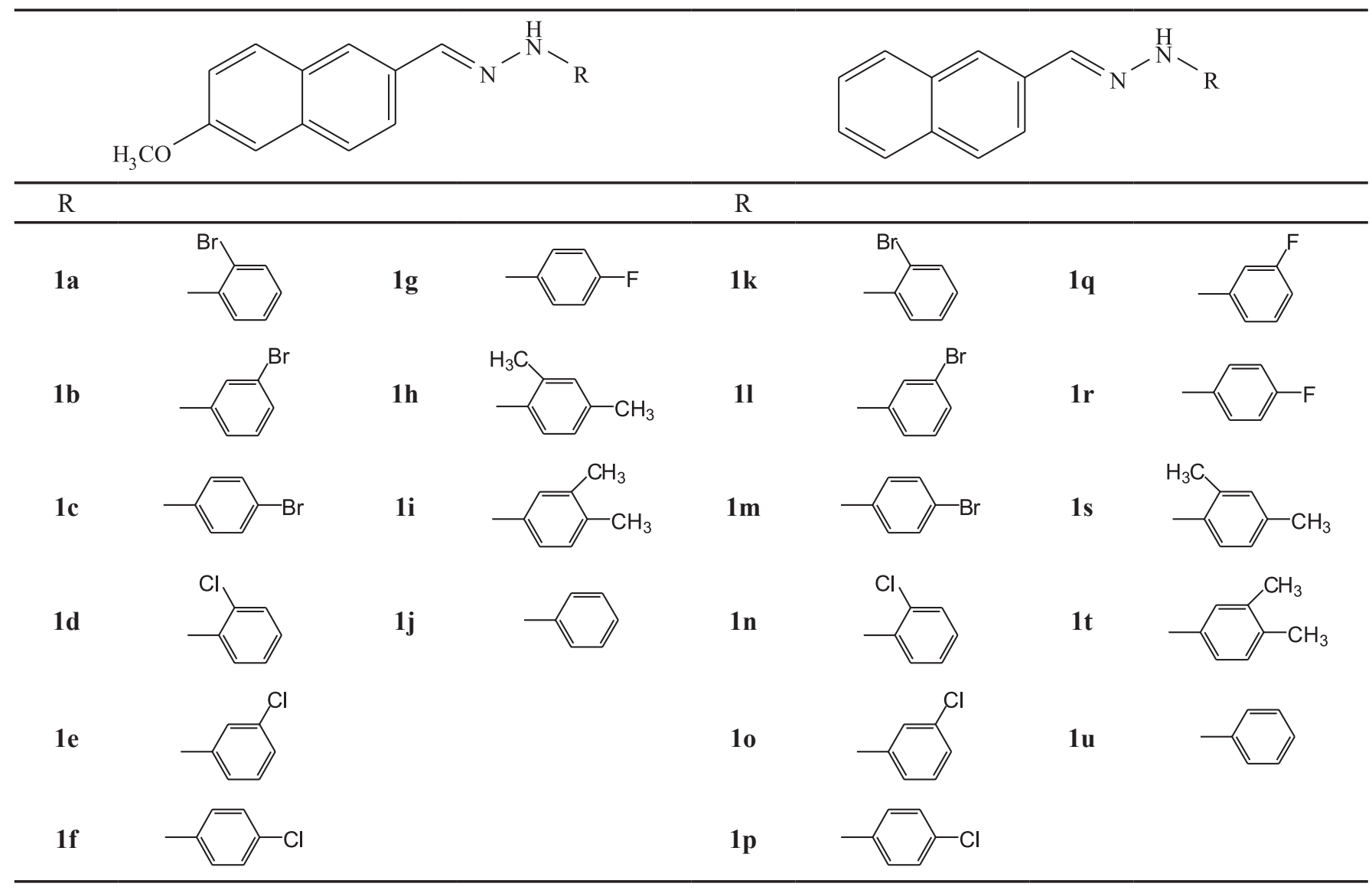

MI). All spectral analyses were performed at the Central Laboratory of the Faculty of Pharmacy at Ankara University. Chromatography was carried out using Merck silica gel 60 (230-400 mesh ASTM). The chemical reagents that were used in synthesis were purchased from Sigma (Germany) and Aldrich (USA).

\section{Chemistry}

The designed compound was synthesized by condensation of phenylhydrazine with 6-methoxy-2naphthaldehyde or 2-naphthaldehyde. All new imines were obtained by using a methodology similar to that in a previous study (Kidwai, Negi, Gupta, 1994). The synthesized compounds were characterized on the basis of ${ }^{1} \mathrm{H}$ and ${ }^{13} \mathrm{C}$ NMR, mass spectra and elemental analyses.

\section{General procedure for the synthesis of compounds 1a-u}

$1 \mathrm{mmol}$ of 6-methoxy-2-naphthaldehyde and 1.3 mmol of phenyl hydrazine hydrochloride or its derivatives were dissolved in absolute ethanol $(20 \mathrm{~mL})$ and heated for $60 \mathrm{~min}$ on a hot water bath in the presence of $\mathrm{CH}_{3} \mathrm{COONa}$ $(0.4 \mathrm{~g})$. After completion of the reaction, the reaction mixture was cooled to room temperature. The precipitate was collected, washed with cold EtOH and recrystallized from $\mathrm{EtOH}$ to achieve 1a-u with 60 to $94 \%$ yield.

1-(2-bromophenyl)-2-((6-methoxynaphthalen-2-yl) methylene)hydrazine (1a)

Yield $94.63 \%$, m.p. $148{ }^{\circ} \mathrm{C} ;{ }^{1} \mathrm{H}-\mathrm{NMR}$ : $\delta 3.93$ (s, 3H, $\left.\mathrm{OCH}_{3}\right) ; 6.73-8.00(\mathrm{~m}, 10 \mathrm{H} \mathrm{ArH}) ; 7.81(\mathrm{~s}, 1 \mathrm{H}, \mathrm{NH}) ; 7.94$ (s, $1 \mathrm{H}, \mathrm{HC}=\mathrm{N}) ;{ }^{13} \mathrm{C}-\mathrm{NMR}: \delta 55.35\left(\mathrm{OCH}_{3}\right), 106.15,106.84$, $114.58,119.14,120.53,123.59,127.02,127.35,128.59$, $128.72,129.67,130.50,132.26,135.05,140.13,141.57$ $(\mathrm{HC}=\mathrm{N}), 158.24:$ MSI MS m/z $355\left(\mathrm{M}^{+}, 90 \%\right), 357(\mathrm{M}+2$, $100 \%$ ). Anal. calcd. for $\mathrm{C}_{18} \mathrm{H}_{15} \mathrm{BrN}_{2} \mathrm{O}: \mathrm{C}, 60.86 \% ; \mathrm{H}$, $4.26 \%$; N, 7.89\%. Found: C, 60.76\%; H, 4.78\%; N, 7.61\%.

1-(3-bromophenyl)-2-((6-methoxynaphthalen-2-yl) methylene)hydrazine (1b)

Yield 86.77\%, m.p. $205{ }^{\circ} \mathrm{C}$; ${ }^{1} \mathrm{H}-\mathrm{NMR}: \delta 3.91$ $\left(\mathrm{s}, 3 \mathrm{H}, \mathrm{OCH}_{3}\right) ; 6.86-7.99(\mathrm{~m}, 10 \mathrm{H} \mathrm{ArH}) ; 7.98(\mathrm{~s}, 1 \mathrm{H}$, 
$\mathrm{HC}=\mathrm{N}) ; 10.52(\mathrm{~s}, 1 \mathrm{H}, \mathrm{NH}) ;{ }^{13} \mathrm{C}-\mathrm{NMR}: \delta 55.22\left(\mathrm{OCH}_{3}\right)$, 106.32, 111.02, 113.98, 118.88, 120.85, 122.45, 123.03, $126.27,127.23,128.38,129.49,130.89,131.03,134.38$, 138.41,146.91 $(\mathrm{C}=\mathrm{N}), 157.63: \mathrm{MSI}$ MS m/z $355\left(\mathrm{M}^{+}, 100\right.$ \%), $357(\mathrm{M}+2100, \%)$. Anal. calcd. for $\mathrm{C}_{18} \mathrm{H}_{15} \mathrm{BrN}_{2} \mathrm{O}$ : C, $60.86 \%$; H, 4.26\%; N, 7.89\%. Found: C, $60.40 \%$; H, $4.31 \% ; \mathrm{N}, 7.83 \%$.

\section{1-(4-bromophenyl)-2-((6-methoxynaphthalen-2-yl) methylene)hydrazine (1c)}

Yield $88.13 \%$, m.p. $198{ }^{\circ} \mathrm{C} ;{ }^{1} \mathrm{H}-\mathrm{NMR}$ : $\delta 3.86$ (s, 3H, $\left.\mathrm{OCH}_{3}\right) ; 7.03-7.92(\mathrm{~m}, 10 \mathrm{H} \mathrm{ArH}) ; 7.97(\mathrm{~s}, 1 \mathrm{H}, \mathrm{HC}=\mathrm{N})$; $10.47(\mathrm{~s}, 1 \mathrm{H}, \mathrm{NH}) ;{ }^{13} \mathrm{C}-\mathrm{NMR}: \delta 55.14$ (O-C), 106.25, 109.26, 113.74, 118.79, 122.93, 125.97, 127.10, 128.33, $129.37,130.99,131.63,134.21,137.70,144.57(\mathrm{C}=\mathrm{N})$, 157.50 (O-C): MSI MS m/z $355\left(\mathrm{M}^{+}, 100 \%\right), 357(\mathrm{M}+2$, $95 \%$ ). Anal. calcd. for $\mathrm{C}_{18} \mathrm{H}_{15} \mathrm{BrN}_{2} \mathrm{O}$ : C, $60.86 \%$; $4.26 \%$; N, 7.89\%. Found: C, 60.78\%; H, 4.37\%; N, 8.04\%.

\section{1-(2-chlorophenyl)-2-((6-methoxynaphthalen-2-yl) methylene)hydrazine (1d)}

Yield $60 \%$, m.p. $148{ }^{\circ} \mathrm{C} ;{ }^{1} \mathrm{H}-\mathrm{NMR}: \delta 3.87$ (s, 3H, $\left.\mathrm{OCH}_{3}\right)$; 6.76-7.94 (m, 10H ArH); $8.39(\mathrm{~s}, 1 \mathrm{H}, \mathrm{HC}=\mathrm{N})$; $9.90(\mathrm{~s}, 1 \mathrm{H}, \mathrm{NH}) ;{ }^{13} \mathrm{C}-\mathrm{NMR}: \delta 55.23\left(\mathrm{OCH}_{3}\right), 106.32$, 114.02, 116.07, 118.89, 119.47, 123.08, 126.48, 127.25, $128.03,128.38,129.32,129.57,130.95,134.49,140.66$, $141.46(\mathrm{C}=\mathrm{N}), 157.69(\mathrm{O}-\mathrm{C}): \mathrm{MSI} \mathrm{MS} \mathrm{m} / \mathrm{z} 311(\mathrm{M}+\mathrm{H}$, $100 \%), 313(\mathrm{M}+2,34 \%)$. Anal. calcd. for $\mathrm{C}_{18} \mathrm{H}_{15} \mathrm{ClN}_{2} \mathrm{O}$ : C, $69.56 \%$; H, 4.86\%; N, 9.01\%. Found: C, 69.16\%; H, $4.90 \% ; \mathrm{N}, 8.96 \%$.

\section{1-(3-chlorophenyl)-2-((6-methoxynaphthalen-2-yl) methylene)hydrazine (1e)}

Yield $64.5 \%$, m.p. $203{ }^{\circ} \mathrm{C}$; ${ }^{1} \mathrm{H}-\mathrm{NMR}$ : $\delta 3.86$ (s, 3H, $\left.\mathrm{OCH}_{3}\right)$; 6.73-7.93 (m, 10H ArH); $7.99(\mathrm{~s}, 1 \mathrm{H}, \mathrm{HC}=\mathrm{N})$; $10.53(\mathrm{~s}, 1 \mathrm{H}, \mathrm{NH}) ;{ }^{13} \mathrm{C}-\mathrm{NMR}: \delta 55.22\left(\mathrm{OCH}_{3}\right), 106.33$, 110.66, 111.14, 117.97, 118.88, 123.05, 126.26, 127.23, $128.39,129.49,130.71,130.92,133.87,134.39,138.36$, $146.81(\mathrm{C}=\mathrm{N}), 157.63(\mathrm{O}-\mathrm{C}):$ MSI MS m/z $311(\mathrm{M}+\mathrm{H}$, $100 \%), 313(\mathrm{M}+2,35 \%)$. Anal. calcd. for $\mathrm{C}_{18} \mathrm{H}_{15} \mathrm{ClN}_{2} \mathrm{O}$ : C, $69.56 \%$; H, 4.86\%; N, 9.01\%. Found: C, 68.96\%; H, $4.92 \% ; \mathrm{N}, 8.94 \%$.

1-(4-chlorophenyl)-2-((6-methoxynaphthalen-2-yl) methylene)hydrazine (1f)

Yield 83.87\%, m.p. $22{ }^{\circ} \mathrm{C} ;{ }^{1} \mathrm{H}-\mathrm{NMR}$ : $\delta 3.86(\mathrm{~s}, 3 \mathrm{H}$, $\left.\mathrm{OCH}_{3}\right)$; 7.07-7.92 (m, 10H ArH); $7.97(\mathrm{~s}, 1 \mathrm{H}, \mathrm{HC}=\mathrm{N})$; $10.46(\mathrm{~s}, 1 \mathrm{H}, \mathrm{NH}) ;{ }^{13} \mathrm{C}-\mathrm{NMR}: \delta 55.21\left(\mathrm{OCH}_{3}\right), 106.32$, 113.34, 118.86, 121.79, 123.01, 126.00, 127.17, 128.42, $128.88,129.44,131.09,134.28,137.67,144.28(\mathrm{C}=\mathrm{N})$, 157.57 (O-C): MSI MS m/z $311(\mathrm{M}+\mathrm{H}, 100 \%), 312$
$(\mathrm{M}+1,32 \%)$. Anal. calcd. for $\mathrm{C}_{18} \mathrm{H}_{15} \mathrm{ClN}_{2} \mathrm{O}: \mathrm{C}, 69.56 \%$; $\mathrm{H}$, $4.86 \%$; N, 9.01\%. Found: C,69.61\%; H, 5.08\%; N, 9.13\%.

\section{1-(4-fluorophenyl)-2-((6-methoxynaphthalen-2-yl) methylene)hydrazine (1g)}

Yield 85.03\%, m.p. $206{ }^{\circ} \mathrm{C} ;{ }^{1} \mathrm{H}-\mathrm{NMR}$ : $\delta 3.85$ (s, 3H, $\left.\mathrm{O} \mathrm{CH}_{3}\right), 7.03-7.92(\mathrm{~m}, 10 \mathrm{H}, \mathrm{Ar}-\mathrm{H}), 7.96(\mathrm{~s}, 1 \mathrm{H}, \mathrm{HC}=\mathrm{N}$ ), 10.32 (s, $1 \mathrm{H}, \mathrm{NH}) ;{ }^{13} \mathrm{C}-\mathrm{NMR}: \delta 55.20\left(\mathrm{OCH}_{3}\right), 106.31$, $112.85,115.48,115.70,118.83,123.01,125.72,127.15$, $128.46,129.39,131.31,134.17,136.88,142.08(\mathrm{C}=\mathrm{N})$, 154.65, 156.97, 157.49 (O-C); MSI MS m/z 295 (M+H, $100 \%), 296\left(\mathrm{M}^{+}+1,28 \%\right)$. Anal. calcd. for $\mathrm{C}_{18} \mathrm{H}_{15} \mathrm{FN}_{2} \mathrm{O}$ : C, 73.45\%; H, 5.14\%; N, 9.52\%. Found: C,73.63\%; H, $5.32 \% ; \mathrm{N}, 9.61 \%$.

\section{1-(2,4-dimethylphenyl)-2-((6-methoxynaphthalen-2-yl)} methylene)hydrazine (1h)

Yield $82.26 \%$, m.p. $169{ }^{\circ} \mathrm{C} ;{ }^{1} \mathrm{H}-\mathrm{NMR}: \delta 2.18$ (s, $\left.6 \mathrm{H}, \mathrm{Ar}-\mathrm{CH}_{3}\right), 3.86\left(\mathrm{~s}, 3 \mathrm{H}, \mathrm{OCH}_{3}\right), 6.86-7.92(\mathrm{~m}, 9 \mathrm{H}$, Ar-H), $8.20(\mathrm{~s}, 1 \mathrm{H}, \mathrm{HC}=\mathrm{N}), 9.44(\mathrm{~s}, 1 \mathrm{H}, \mathrm{NH}) ;{ }^{13} \mathrm{C}-\mathrm{NMR}$ : $\delta 17.37\left(\mathrm{Ar}-\mathrm{CH}_{3}\right), 20.11 ;\left(\mathrm{Ar}-\mathrm{CH}_{3}\right), 55.17\left(\mathrm{OCH}_{3}\right)$, 106.30, 112.23, 118.74, 120.60, 123.04, 125.49, 127.06, 127.12, 128.47, 129.36, 130.88, 131.54, 134.11, 137.67, $141.00(\mathrm{C}=\mathrm{N}), 157.41(\mathrm{O}-\mathrm{C})$; MSI MS m/z $305(\mathrm{M}+\mathrm{H}$, $100 \%), 306\left(\mathrm{M}^{+}+1,32 \%\right)$. Anal. calcd. for $\mathrm{C}_{20} \mathrm{H}_{20} \mathrm{~N}_{2} \mathrm{O}$ : C, 78.92\%; H, 6.62\%; N, 9.20\%. Found: C,78.39\%; H, $6.76 \% ; \mathrm{N}, 8.77 \%$.

\section{1-(3,4-dimethylphenyl)-2-((6-methoxynaphthalen-2-yl) methylene)hydrazine (1i)}

Yield $83.87 \%$, m.p. $230{ }^{\circ} \mathrm{C} ;{ }^{1} \mathrm{H}-\mathrm{NMR}: \delta 2.11$ ( s, $3 \mathrm{H}$ , Ar- $\left.\mathrm{CH}_{3}\right), 2.18\left(\mathrm{~s}, 3 \mathrm{H}, \mathrm{Ar}-\mathrm{CH}_{3}\right), 3.85\left(\mathrm{~s}, 3 \mathrm{H}, \mathrm{OCH}_{3}\right)$, 6.80-7.90 ( $\mathrm{m}, 9 \mathrm{H}, \mathrm{Ar}-\mathrm{H}), 7.92(\mathrm{~s}, 1 \mathrm{H}, \mathrm{HC}=\mathrm{N}), 10.14$ ( $\mathrm{s}, 1 \mathrm{H}, \mathrm{NH}) ;{ }^{13} \mathrm{C}-\mathrm{NMR}: 18.55\left(\mathrm{Ar}-\mathrm{CH}_{3}\right), 19.76\left(\mathrm{Ar}-\mathrm{CH}_{3}\right.$ ), $55.19\left(\mathrm{OCH}_{3}\right), 106.31,109.48,113.30,118.78,123.02$, $125.41,125.99,127.12,128.50,129.34,130.03,131.56$, 134.04, 135.95, 136.62, $143.39(\mathrm{C}=\mathrm{N}), 157.39(\mathrm{O}-\mathrm{C})$; MSI MS m/z $305(\mathrm{M}+\mathrm{H}, 100 \%), 306\left(\mathrm{M}^{+}+1,65 \%\right)$. Anal. calcd. for $\mathrm{C}_{20} \mathrm{H}_{20} \mathrm{~N}_{2} \mathrm{O}$ : C, 78.92\%; H, 6.62\%; N, 9.20\%. Found: C,78.42\%; H, 6.46\%; N, 9.18\%.

\section{1-((6-methoxynaphthalen-2-yl)methylene)-2- phenylhydrazine (1j)}

Yield 90.68\%, m.p. $229^{\circ} \mathrm{C} ;{ }^{1} \mathrm{H}-\mathrm{NMR}: \delta 3.86$ (s, 3H, $\left.\mathrm{OCH}_{3}\right)$; 6.72-7.93 (m, 11H ArH); $7.97(\mathrm{~s}, 1 \mathrm{H}, \mathrm{HC}=\mathrm{N})$; $10.29(\mathrm{~s}, 1 \mathrm{H}, \mathrm{NH}) ;{ }^{13} \mathrm{C}-\mathrm{NMR}: \delta 55.20\left(\mathrm{OCH}_{3}\right), 106.31$, 111.94, 118.60, 118.82, 123.03, 125.69, 127.15, 128.46, $129.10,129.40,131.38,134.16,136.79,145.40(\mathrm{C}=\mathrm{N})$, 157.47(O-C): MSI MS m/z $277(\mathrm{M}+\mathrm{H}, 100 \%)$. Anal. calcd. for $\mathrm{C}_{18} \mathrm{H}_{16} \mathrm{~N}_{2} \mathrm{O}$ : C, 78.24\%; H, 5.84\%; N, 10.14\%. Found: C,77.99\%; H, 5.84\%; N, $10.10 \%$. 


\section{1-(2-bromophenyl)-2-(naphthalen-2-ylmethylene) hydrazine (1k)}

Yield $83.10 \%$, m.p. $163{ }^{\circ} \mathrm{C} ;{ }^{1} \mathrm{H}-\mathrm{NMR}: \delta 6.72-8.02$ $(\mathrm{m}, 11 \mathrm{H} \mathrm{ArH}) ; 8.46(\mathrm{~s}, 1 \mathrm{H}, \mathrm{HC}=\mathrm{N}) ; 9.77(\mathrm{~s}, 1 \mathrm{H}, \mathrm{NH})$ ; ${ }^{13} \mathrm{C}-\mathrm{NMR}: \delta 106.10,114.63,120.40,122.54,126.35$, $126.56,126.60,127.70,128.00,128.30,128.60,132.59$, 133.07, 133.09, 133.19, 140.52, $142.39(\mathrm{C}=\mathrm{N})$ : MSI MS $\mathrm{m} / \mathrm{z} 325\left(\mathrm{M}^{+}, 100 \%\right), 327(\mathrm{M}+2,100 \%)$. Anal. calcd. for $\mathrm{C}_{17} \mathrm{H}_{13} \mathrm{BrN}_{2}$ : C, $62.79 \% ; \mathrm{H}, 4.03 \%$; N, 8.61\%. Found: $\mathrm{C}, 62.61 \% ; \mathrm{H}, 4.13 \%$; N, 8.79\%.

\section{1-(3-bromophenyl)-2-(naphthalen-2-ylmethylene) hydrazine (1)}

Yield $75.40 \%$, m.p. $175{ }^{\circ} \mathrm{C}$; ${ }^{1} \mathrm{H}-\mathrm{NMR}: \delta 6.88-7.98$ (m, 11H ArH); $8.03(\mathrm{~s}, 1 \mathrm{H}, \mathrm{HC}=\mathrm{N}) ; 10.62(\mathrm{~s}, 1 \mathrm{H}, \mathrm{NH})$ ; ${ }^{13} \mathrm{C}-\mathrm{NMR}: \delta 111.13,114.12,121.10,122.47,122.49$, $126.25,126.35,126.53,127.69,127.91,128.26,131.06$, $132.95,133.10,133.14,138.09,146.78(\mathrm{C}=\mathrm{N})$ : MSI MS m/z $325\left(\mathrm{M}^{+}, 95 \%\right), 327(\mathrm{M}+2,100 \%)$. Anal. calcd. for $\mathrm{C}_{17} \mathrm{H}_{13} \mathrm{BrN}_{2}$ : C, 62.79\%; H, 4.03\%; N, 8.61\%. Found: $\mathrm{C}, 62.46 \% ; \mathrm{H}, 4.09 \%$;, $8.75 \%$.

\section{1-(4-bromophenyl)-2-(naphthalen-2-ylmethylene) hydrazine (1m)}

Yield 65.86\%, m.p. $218{ }^{\circ} \mathrm{C} ;{ }^{1} \mathrm{H}-\mathrm{NMR}: \delta$ 7.05-7.97 $(\mathrm{m}, 11 \mathrm{H} \mathrm{ArH}) ; 8.02(\mathrm{~s}, 1 \mathrm{H}, \mathrm{HC}=\mathrm{N}) ; 10.58(\mathrm{~s}, 1 \mathrm{H}, \mathrm{NH})$ ; ${ }^{13} \mathrm{C}-\mathrm{NMR}: \delta 109.68,113.97,122.46,126.11,126.17$, $126.53,127.68,127.86,128.21,131.74,132.88,133.13$, 133.30, 137.45, $144.51(\mathrm{C}=\mathrm{N}):$ MSI MS m/z $325\left(\mathrm{M}^{+}\right.$, $93 \%), 327(\mathrm{M}+2,100 \%)$. Anal. calcd. for $\mathrm{C}_{17} \mathrm{H}_{13} \mathrm{BrN}_{2}$ : C, 62.79\%; H, 4.03\%; N, 8.61\%. Found: C, 62.03\%; H, $4.11 \%$; N, $8.67 \%$.

\section{1-(2-chlorophenyl)-2-(naphthalen-2-ylmethylene) hydrazine (1n)}

Yield 85.71\%, m.p. $168{ }^{\circ} \mathrm{C} ;{ }^{1} \mathrm{H}-\mathrm{NMR}: \delta 6.77-8.01$ (m, 11H ArH); $8.43(\mathrm{~s}, 1 \mathrm{H}, \mathrm{HC}=\mathrm{N}) ; 10.01(\mathrm{~s}, 1 \mathrm{H}, \mathrm{NH})$ ; ${ }^{13} \mathrm{C}-\mathrm{NMR}: \delta 114.13,116.20,119.69,122.51,126.34$, $126.55,126.57,127.68,127.98,128.05,128.30,129.36$, 133.06, 133.10, 133.20,140.33, $141.35(\mathrm{C}=\mathrm{N})$ : MSI MS m/z $281(\mathrm{M}+\mathrm{H}, 100 \%), 283\left(\mathrm{M}^{+}+2,34 \%\right)$. Anal. calcd. for $\mathrm{C}_{17} \mathrm{H}_{13} \mathrm{ClN}_{2}$ : C, 72.73\%; H, 4.67\%; N, 9.98\%. Found: $\mathrm{C}, 72.65 \% ; \mathrm{H}, 4.76 \% ; \mathrm{N}, 10.02 \%$.

\section{1-(3-chlorophenyl)-2-(naphthalen-2-ylmethylene) hydrazine (1o)}

Yield 82.14\%, m.p. $172{ }^{\circ} \mathrm{C} ;{ }^{1} \mathrm{H}-\mathrm{NMR}: \delta 6.75-7.99$ (m, 11H ArH); $8.03(\mathrm{~s}, 1 \mathrm{H}, \mathrm{HC}=\mathrm{N}) ; 10.63(\mathrm{~s}, 1 \mathrm{H}, \mathrm{NH})$ ; ${ }^{13} \mathrm{C}-\mathrm{NMR}: \delta 111.24,111.72,118.68,122.98,126.73$, $126.81,127.02,128.17,128.39,128.74,131.24,133.43$,
133.58, 133.62, 134.35, 138.51, $147.14(\mathrm{C}=\mathrm{N})$ : MSI MS m/z $281(\mathrm{M}+\mathrm{H}, 100 \%), 283\left(\mathrm{M}^{+}+2,32 \%\right)$. Anal. calcd. for $\mathrm{C}_{17} \mathrm{H}_{13} \mathrm{ClN}_{2}$ : C, $72.73 \%$; H, 4.67\%; N, 9.98\%. Found: $\mathrm{C}, 72.03 \% ; \mathrm{H}, 4.73 \%$; N, 9.88\%.

\section{1-(4-chlorophenyl)-2-(naphthalen-2-ylmethylene)} hydrazine (1p)

Yield 85\%, m.p. $223{ }^{\circ} \mathrm{C} ;{ }^{1} \mathrm{H}-\mathrm{NMR}: \delta$ 7.07-7.97 $(\mathrm{m}, 11 \mathrm{H} \mathrm{ArH}) ; 8.01(\mathrm{~s}, 1 \mathrm{H}, \mathrm{HC}=\mathrm{N}) ; 10.57(\mathrm{~s}, 1 \mathrm{H}, \mathrm{NH})$ ; ${ }^{13} \mathrm{C}-\mathrm{NMR}: \delta 113.46,122.04,122.47,126.07,126.16$, $126.53,127.69,128.86,128.21,128.92,132.88,133.14$, 133.33, 137.35, $144.15(\mathrm{C}=\mathrm{N})$ : MSI MS m/z $281(\mathrm{M}+\mathrm{H}$, $100 \%), 283\left(\mathrm{M}^{+}+2,35 \%\right)$. Anal. calcd. for $\mathrm{C}_{17} \mathrm{H}_{13} \mathrm{ClN}_{2}$ : C, 72.73\%; H, 4.67\%; N, 9.98\%. Found: C,73.08\%; H, $4.89 \%$; N $10.11 \%$.

\section{1-(3-fluorophenyl)-2-(naphthalen-2-ylmethylene)} hydrazine (1q)

Yield 77\%, m.p. $200{ }^{\circ} \mathrm{C} ;{ }^{1} \mathrm{H}-\mathrm{NMR}: \delta 6.50-7.99$ (m, 11H, Ar-H), $8.03(\mathrm{~s}, 1 \mathrm{H}, \mathrm{HC}=\mathrm{N}), 10.65(\mathrm{~s}, 1 \mathrm{H}, \mathrm{NH})$; ${ }^{13}$ C-NMR: $\delta$ 98.54, 104.78, 104.99, 108.19, 122.54, 126.24, 459.53, 127.69, 127.90, 128.24, 130.70, 132.94, 133.16, 137.76, $147.23(\mathrm{C}=\mathrm{N}), 162.19,164.58$; MSI MS $\mathrm{m} / \mathrm{z} 265(\mathrm{M}+\mathrm{H}, 100 \%), 266\left(\mathrm{M}^{+}+1,24 \%\right)$. Anal. calcd. for C17H13FN2: C, 77.25\%; H, 4.96\%; N, 10.60\%. Found: C,76.91\%; H, 5.04\%; N, 10.62\%.

\section{1-(4-fluorophenyl)-2-(naphthalen-2-ylmethylene)} hydrazine (1r)

Yield $90.90 \%$, m.p. $232{ }^{\circ} \mathrm{C} ;{ }^{1} \mathrm{H}-\mathrm{NMR}: \delta$ 7.04-7.98 $(\mathrm{m}, 11 \mathrm{H}, \mathrm{Ar}-\mathrm{H}), 8.00(\mathrm{~s}, 1 \mathrm{H}, \mathrm{HC}=\mathrm{N}), 10.43(\mathrm{~s}, 1 \mathrm{H}, \mathrm{NH})$; ${ }^{13} \mathrm{C}$-NMR: $\delta 112.99,115.75,122.47,125.77,126.50$, $127.67,127.81,128.17,132.79,133.17,136.53,141.91$ $(\mathrm{C}=\mathrm{N}), 154.77,157.09 ; \mathrm{MSI}$ MS m/z $265(\mathrm{M}+\mathrm{H}, 100 \%)$, $266\left(\mathrm{M}^{+}+1,30 \%\right)$. Anal. calcd. for $\mathrm{C}_{17} \mathrm{H}_{13} \mathrm{FN}_{2}$ : C, 77.25\%; H, 4.96\%; N, 10.60\%. Found: C,76.01\%; H, 5.01\%; N, $10.79 \%$.

\section{1-(2,4-dimethylphenyl)-2-(naphthalen-2-ylmethylene) hydrazine (1s)}

Yield $85.71 \%$, m.p. $178{ }^{\circ} \mathrm{C} ;{ }^{1} \mathrm{H}-\mathrm{NMR}: \delta 2.18(\mathrm{~s}, 3 \mathrm{H}$, $\left.\mathrm{Ar}-\mathrm{CH}_{3}\right), 2.20\left(\mathrm{~s}, 3 \mathrm{H}, \mathrm{Ar}-\mathrm{CH}_{3}\right), 6.87-7.98(\mathrm{~m}, 10 \mathrm{H}, \mathrm{ArH}$ ), $8.24(\mathrm{~s}, 1 \mathrm{H}, \mathrm{HC}=\mathrm{N}), 9.57(\mathrm{~s}, 1 \mathrm{H}, \mathrm{NH}) ;{ }^{13} \mathrm{C}-\mathrm{NMR}: \delta$ $17.43\left(\mathrm{Ar}-\mathrm{CH}_{3}\right), 20.17\left(\mathrm{Ar}-\mathrm{CH}_{3}\right), 112.37,120.76,122.52$, $125.58,125.98,126.47,127.14,127.40,127.66,127.82$, $128.19,130.96,132.77,133.23,133.81,137.33,140.88$ $(\mathrm{C}=\mathrm{N}) ; \mathrm{MSI} \mathrm{MS} \mathrm{m} / \mathrm{z} 275(\mathrm{M}+\mathrm{H}, 100 \%)$. Anal. calcd. for $\mathrm{C}_{19} \mathrm{H}_{18} \mathrm{~N}_{2}$ : C, 83.18\%; H, 6.61\%; N, 10.21\%. Found: $\mathrm{C}, 82.58 \% ; \mathrm{H}, 6.64 \%$; N $10.14 \%$. 
1-(3,4-dimethylphenyl)-2-(naphthalen-2-ylmethylene) hydrazine (1t).

Yield 78.57\%, m.p. $218^{\circ} \mathrm{C} ;{ }^{1} \mathrm{H}-\mathrm{NMR}: \delta 2.12(\mathrm{~s}, 3 \mathrm{H}$, Ar- $\left.\mathrm{CH}_{3}\right), 2.18\left(\mathrm{~s}, 3 \mathrm{H}, \mathrm{Ar}-\mathrm{CH}_{3}\right), 6.82-7.98(\mathrm{~m}, 10 \mathrm{H}, \mathrm{Ar}-$ $\mathrm{H}), 7.85(\mathrm{~s}, 1 \mathrm{H}, \mathrm{HC}=\mathrm{N}), 10.25(\mathrm{~s}, 1 \mathrm{H}, \mathrm{NH}) ;{ }^{13} \mathrm{C}-\mathrm{NMR}$ : $\delta 18.55\left(\mathrm{Ar}-\mathrm{CH}_{3}\right), 19.76\left(\mathrm{Ar}-\mathrm{CH}_{3}\right), 109.57,113.38$, $122.48,125.42,125.88,126.22,126.43,127.65,127.75$, $128.11,130.04,132.66,133.22,133.78,135.58,136.65$, $143.22(\mathrm{C}=\mathrm{N})$; MSI MS m/z $275(\mathrm{M}+\mathrm{H}, 100 \%)$. Anal. calcd. for $\mathrm{C}_{19} \mathrm{H}_{18} \mathrm{~N}_{2}$ : C, 83.18\%; H, 6.61\%; N, $10.21 \%$. Found: C, $82.90 \%$; H, $6.63 \%$; N , 10.18\%.

\section{1-(naphthalen-2-ylmethylene)-2-phenylhydrazine (1u)}

Yield 79.67\%, m.p. $223{ }^{\circ} \mathrm{C} ;{ }^{1} \mathrm{H}-\mathrm{NMR}$ : $\delta$ 6.73-7.98 $(\mathrm{m}, 12 \mathrm{H} \mathrm{ArH}) ; 8.01(\mathrm{~s}, 1 \mathrm{H}, \mathrm{HC}=\mathrm{N}) ; 10.44(\mathrm{~s}, 1 \mathrm{H}, \mathrm{NH})$ ; ${ }^{13} \mathrm{C}-\mathrm{NMR}: \delta 112.04,118.82,122.48,125.74,126.02$, $126.48,127.67,127.81,128.17,129.12,132.77,133.18$, 133.61, 136.44, $145.21(\mathrm{C}=\mathrm{N}):$ MSI MS m/z $247(\mathrm{M}+\mathrm{H}$, $100 \%$ ). Anal. calcd. for $\mathrm{C}_{17} \mathrm{H}_{14} \mathrm{~N}_{2}: \mathrm{C}, 82.90 \%$; H, 5.733\%; N, $11.37 \%$. Found: C, $82.30 \% ; H, 5.79 \%$; N $11.29 \%$. All the experimental and characterization data of $\mathbf{1} \mathbf{u}$ are in agreement with the literature. (Weil, Ostermeier, 1921)

\section{EXPERIMENTAL - BIOLOGICAL ACTIVITY}

\section{Cell culture and chemicals}

The CHO-K1 cell line (ATCC) was maintained with Dulbecco's Modified Eagle Serum/F12 Ham (DMEM: F12) which was supplemented with $10 \%$ fetal bovine serum (FBS) and $1 \mathrm{mM}$ sodium pyruvate at $37^{\circ} \mathrm{C}$ and in a humidified atmosphere containing $5 \% \mathrm{CO}_{2}$. Cell culture media and assay chemicals were obtained from SigmaAldrich (St. Louis, MO) except for FBS (Gibco, Grand Island, NY) and sodium pyruvate (Santa Cruz Biotech. Inc., TX).

\section{Free radical scavenging activity evaluation with DPPH assay}

2,2-diphenyl-1-picrylhydrazyl (DPPH) is a stable free radical with a maximum absorbance of $520 \mathrm{~nm}$. DPPH is reduced with the absence of an antioxidant molecule and loses its reactivity. Thereby, the solution with a dark blue color becomes colorless (Blois, 1958). The DPPH assay was performed just as our previous protocol (Carocci et al., 2014). The novel compounds were added into $150 \mu \mathrm{L}$ of DPPH methanol: water $(4: 1)$ solution with final concentrations of $10 \mu \mathrm{M}$ and $100 \mu \mathrm{M}$ in 96-well plates. DPPH reduction was monitored in a microplate reader at a wavelength of $517 \mathrm{~nm}$ for 60 minutes. The radical scavenging activities of the molecules (RSA) were calculated as:

$$
\mathrm{RSA} \%=((\mathrm{A} 0-\mathrm{A} 1) / \mathrm{A} 0 \times 100)
$$

A0: absorbance value of solvent control - DPPH solution containing $10 \%$ DMSO - A1: molecular absorbance value - $180 \mu \mathrm{L}$ DPPH solution containing $20 \mu \mathrm{L}$ molecule of DMSO solution.

\section{Cytotoxicity evaluation with MTT assay}

MTT (3-(4,5-dimethylthiazol-2-yl)-2,5diphenyltetrazolium bromide) is a water-soluble tetrazolium salt. MTT is reduced to purple formazan crystals by the mitochondrial succinate dehydrogenase enzyme. Formazan crystals are dissolved in the appropriate solvent, and the optical density is measured in a spectrophotometer (Niles, Moravec, Riss, 2008).

$\mathrm{CHO}-\mathrm{K} 1$ cells were plated at a concentration of 5000 cells / well in 96-well plates. The cells were incubated for 24 hours in a humidified environment containing $5 \% \mathrm{CO}_{2}$ at $37{ }^{\circ} \mathrm{C}$. The final concentrations of the test substances were added to the cell medium at $10 \mu \mathrm{M}$, and incubation was carried out with the test substances for 24 hours. Control (medium), vehicle control (0.1\% DMSO) and positive control $(171 \mu \mathrm{M}$ Triton X-100) groups were used in all experiments. After exposure to the test substances, the media were removed, and the wells were washed with phosphatebuffered saline (PBS). The wells which contained the cells were incubated for 4 hours in a MTT solution with a final concentration of $1 \mathrm{mg} / \mathrm{mL}$ in the medium (Mossman, 1983). After incubation, the MTT solution was removed from the wells, and the formazan crystals were dissolved in $150 \mu \mathrm{L}$ of DMSO. Absorbance values were recorded in a Thermo / Varioskan Flash microplate reader at a wavelength of $550 \mathrm{~nm}$. The effect of the novel compounds on cell viability was calculated as \% viability in comparison to the vehicle control (the viability of the vehicle control was accepted as $100 \%$ ).

\section{Statistical analyses}

The statistical analyses of the DPPH and MTT experiments were carried out by using the parametric method of "student's t-test". 


\section{RESULTS}

\section{Antioxidant activities of novel compounds}

The radical scavenging activities of the novel compounds were investigated by the DPPH assay at final concentrations of $10 \mu \mathrm{M}$ and $100 \mu \mathrm{M}$ (Figure 1). According to these results, all molecules exhibited statistically significant radical scavenging activity in comparison to the vehicle control.

\section{Effects on cell viability of novel compounds}

The effects of the molecules on cell viability were demonstrated by the MTT assay. The antiproliferative effects of the molecules were investigated at the final concentration of $10 \mu \mathrm{M}$ in $0.1 \%$ DMSO (Figure 3).

\section{DISCUSSION AND CONCLUSION}

Biological processes such as aging are thought to play a role in oxidative stress in the pathology of many diseases such as diabetes, atherosclerosis, rheumatoid arthritis and cancer. Therefore, external antioxidant supplementation and/or strengthening of the body's antioxidant defense system are proposed as treatment approaches for these diseases. One of the mechanisms of action of antioxidant molecules is cleaning/scavenging of reactive oxygen derivatives. In this study, novel compounds were synthesized by bioisosteric modification of the indole ring of MLT, and the possible antioxidant potentials of these compounds were studied by investigating their radical scavenging activities. The radical scavenging activities of the 1-(halogenated phenyl)-2-((6-methoxynaphthalen2-yl)methylene)hydrazine derivatives (1a-j) were higher than those of the 1-(halogenated phenyl)-2-naphthalen-2yl)methylene)hydrazine (1 k-u) derivatives. It is believed that the 6-methoxy substitution process in these halogenbearing derivatives may have increased their activity. In the halogen-free derivatives, methoxy substitution $(\mathbf{1 i}, \mathbf{1} \mathbf{j})$ was not found to cause an increase in activity in comparison to the non-methoxy-substituted derivatives $(1 \mathbf{t}, 1 \mathbf{u})$. The presence of halogen in the $o$-position reduced the radical scavenging activity $(\mathbf{1 a}, \mathbf{1 d}, \mathbf{1 1} \& \mathbf{1 0})$, but $m$ and $p$-halogen substitution increased the activity of the phenyl substitution in the hydrazine group.

One of the factors causing the greatest time, cost and labor loss in drug discovery and development studies is

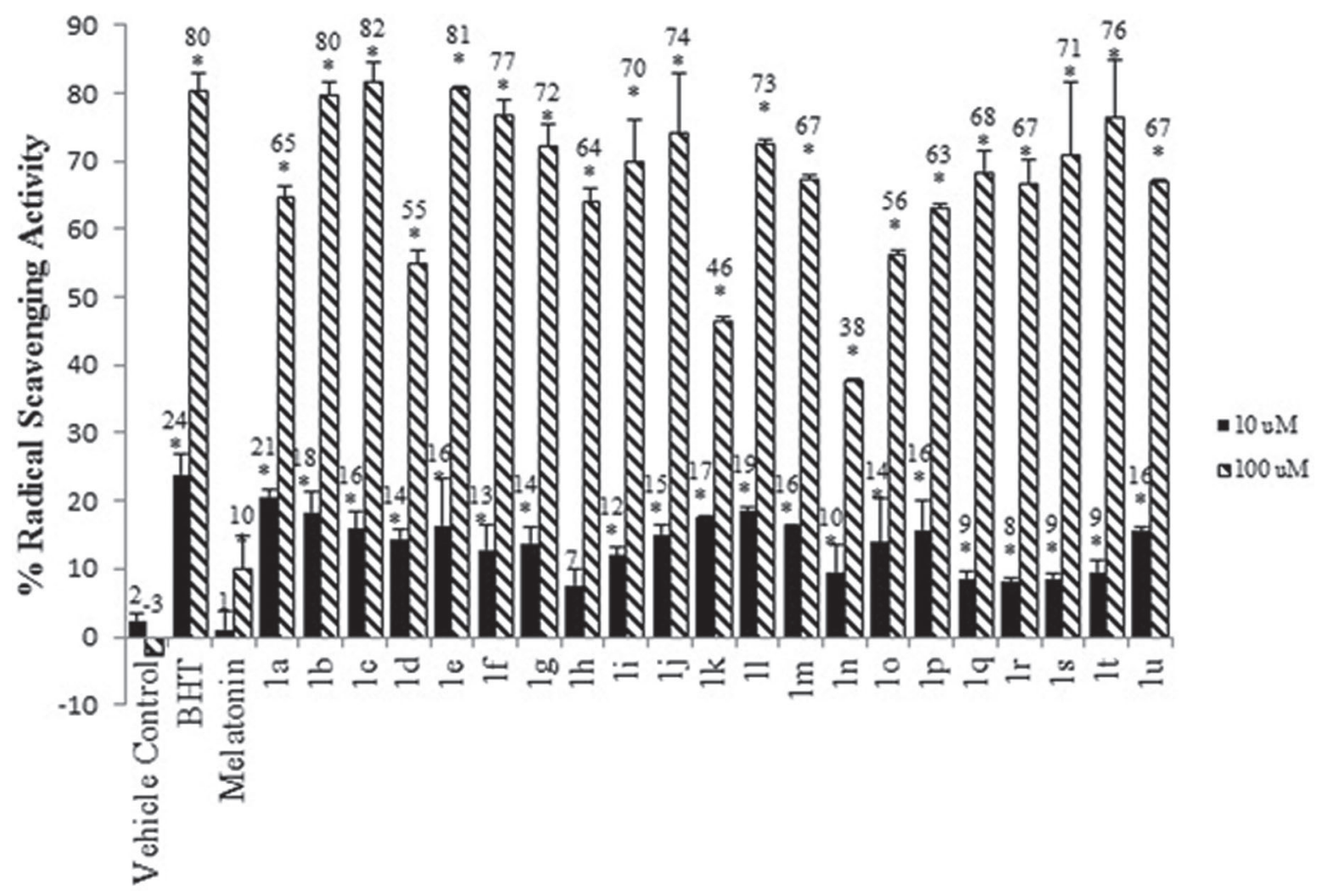

FIGURE 2 - Radical scavenging activities of the novel compounds according to the DPPH assay. Bars represent "\% RSA Mean \pm standard deviation". Melatonin (MLT) and butylhydroxytoluene (BHT) were used as reference compounds. The results were obtained by studying three different groups for each molecule. Statistically significant values $(p<0,05)$ were expressed with "*”. 


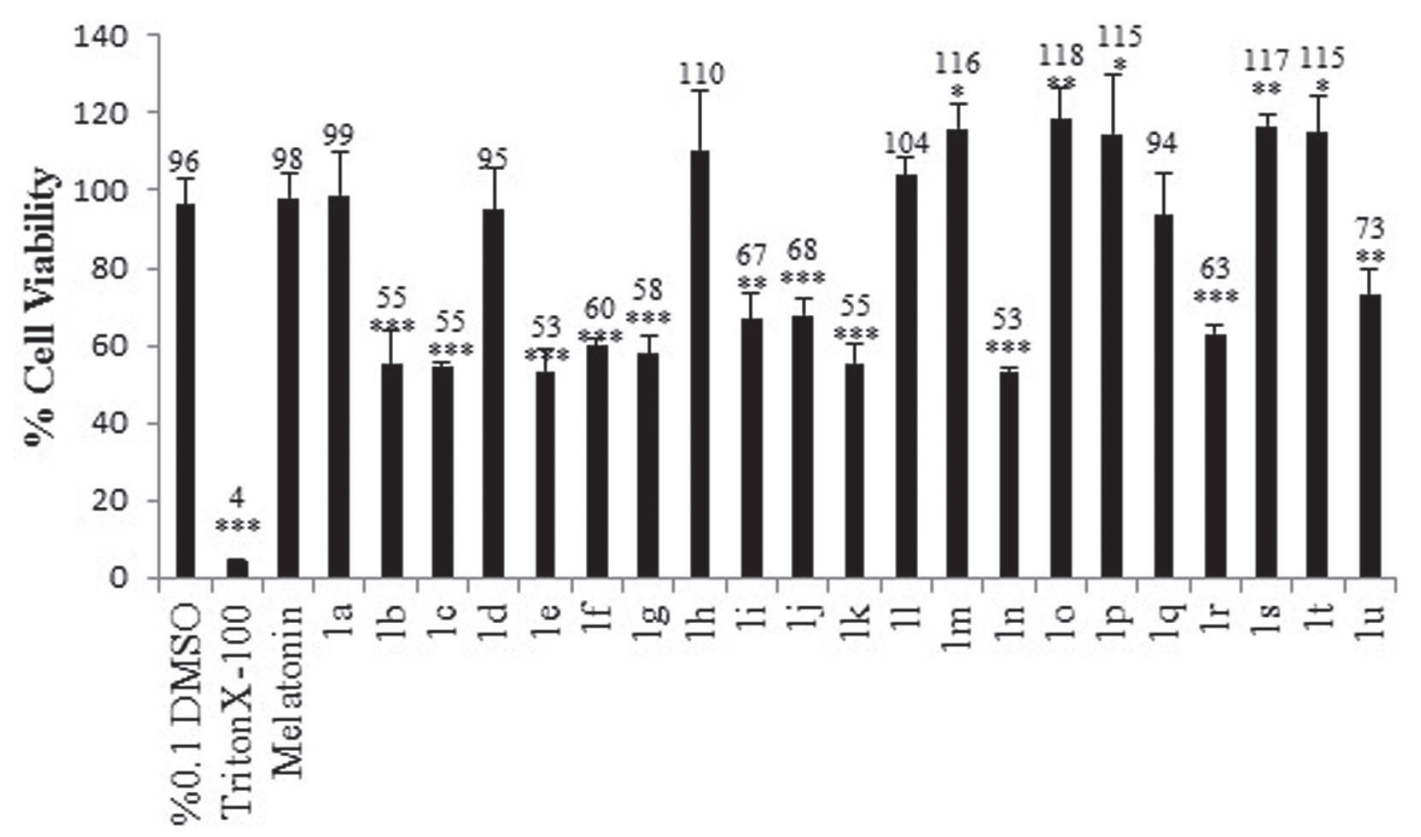

FIGURE 3 - Effects of novel compounds on cell viability in CHO cells. Bars represent the "\% cell viability mean \pm standart deviation" values from 3 separate experiments for each molecule. \% Viability evaluation was calculated according to solvent control (DMSO $0.1 \%$ ). Triton $\mathrm{X}-100$ was used as a positive control. Statistical significance was expressed as $* \mathrm{p}<0,05 ; * * \mathrm{p}<0.01 ; * * * \mathrm{p}<0.005$.

that a candidate compound that is found at the late stages of studies might be thrown away as all work is finally done, so it is a possibility that the toxic effects of such a candidate compound are not studied. For this reason, the pharmaceutical industry and regulatory agencies recommend in vitro rapid screening tests to be conducted at the early stages of drug discovery and development studies, and accordingly, investigation of the cytotoxicity of such substances will prevent these losses. In the light of these information and proposals, the possible cytotoxic effects of the novel compounds were investigated in a healthy cell line by the MTT assay in our study. The MTT assay detects the viable cells with active metabolism which convert MTT into a purple colored formazan product. Thus, color formation serves as a convenient marker of only the viable cells. The findings indicated that there was no significant structure-activity relationship in the potentially cytotoxic effects of the substances, but the 6-methoxy naphthalene compounds seemed to be more cytotoxic than the non-methoxy naphthalene compounds. Moreover, the ortho halogenation on the phenyl ring was found to decrease the cytotoxic potential of the compounds in general. Some substances that are found to have radical scavenging effects appear to have cytotoxic potency. For this reason, it is recommended to evaluate and confirm the radical scavenging/antioxidant effects in a cell-based in vitro model and determine the cytotoxicity potential of the substances at their effective concentrations. It is also possible to investigate the mechanisms of their cytotoxic effects by using a battery of cytotoxicity assays based on various mechanisms instead of a sole assay, MTT.

\section{Z,E-isomerism evaluation of the synthesized compounds}

Molecules that have the hydrazono group with other functional groups have some special physical and chemical properties. In most cases, these compounds can exist as either anti- $E$ or syn- $Z$ isomers. The ${ }^{1} \mathrm{H},{ }^{13} \mathrm{C}$ and ${ }^{15} \mathrm{~N}$ NMR spectroscopic techniques and an X-ray diffraction of single crystals are much more informative tools for the study of the fine structure of hydrazono-compounds including information on $Z, E$-isomerism and hydrogen bonding.

Studies in the literature with compounds that are structurally similar to naphthalene-hydrazone derivatives showed that these molecules exist as anti- $E$ isomers (Figure 4). The structure of 2-thiophenecarbaldehyde 2-quinolylhydrazone (A) was investigated by Mague et al. (1997) using X-ray Crystallographic methods, and the molecule was found in the anti- $E$ isomer form. Based on our previous study (Yilmaz, Coban, Suzen, 2011), we observed that the twenty-one synthesized indole hydrazide/hydrazone (B) derivatives (except for one derivative) existed as anti- $E$ isomers. Our research on quinolone hydrazine derivatives showed similar results (Puskullu et al., 2015). A series of quinoline-2carboxaldehydes hydrazones was synthesized (C) and 
<smiles></smiles>

(A)<smiles>C(=N/Nc1ccccc1)\c1ccc2ccccc2n1</smiles>

(C)

FIGURE 4 - Z,E-isomerism of some hydrazono-compounds

characterized. None of the compounds showed syn- $\mathrm{Z}$ isomers according to the findings of the ${ }^{1} \mathrm{H},{ }^{13} \mathrm{C}$ and ${ }^{15} \mathrm{~N}$ NMR spectroscopic analyses. In this study, we observed similar results with naphthalene derivatives (D). No signals were detected to represent isomerism by the ${ }^{1} \mathrm{H},{ }^{13} \mathrm{C}$ NMR analyses. These literature data and reports (Soltani Rad, Khalafi-Nezhad, Behrouz, 2010), prove that all of the synthesized compounds are anti-E isomer.

\section{ACKNOWLEDGEMENTS}

The authors appreciate the Scientific Research Council of Erzincan Binali Yildirim University for their support of the Project (Project No: SAG-A-240215-0120).

\section{REFERENCES}

Aguiar CC, Almeida AB, Araujo PV, Vasconcelos GS, Chaves EM, Do Vale OC, et al. Effect of agomelatine on oxidative stress in the brain of mice after chemically induced seizures. Cell Mol Neurobiol. 2013;33(6):825-835.

Allegra M, Reiter RJ, Tan DX, Gentile C, Tesoriere L, Livrea MA. The chemistry of melatonin's interaction with reactive species. J Pineal Res. 2003;34(1):1-10.

Azim MS, Agarwal NB, Vohora D. Effects of agomelatine on pentylenetetrazole-induced kindling, kindling-associated oxidative stress, and behavioral despair in mice and modulation of its actions by luzindole and 1-(m-chlorophenyl) piperazine. Epilepsy Behav. 2017;72:140-144.<smiles>[R]NN=Cc1c[nH]c2ccc(Cl)cc12</smiles>

(B)<smiles>[R]N/[N+]([O-])=C/c1ccc2cc(OC)ccc2c1</smiles>

(D)

Blois MS. Antioxidant determinations by the use of a stable free radical. Nature. 1958;181:1199-1200.

Carocci A, Catalano A, Sinicropi MS. Melatonergic drugs in development. Clin Pharmacol. 2014;6:127-137.

Danilov A, Kurganova J. Melatonin in chronic pain syndromes. Pain Ther. 2016;5(1):1-17.

De Mello AH, Souza Lda R, Cereja AC, Schraiber Rde B, Florentino D, Martins MM, et al. Effect of subchronic administration of agomelatine on brain energy metabolism and oxidative stress parameters in rats. Psychiatry Clin Neurosci. 2016;70(4):159-166.

Ding Y, Li H, Meng Y, Zhang T, Li J, Chen OY, et al. Direct synthesis of hydrazones by visible light mediated aerobic oxidative cleavage of the $\mathrm{C}=\mathrm{C}$ bond. Organic Chemistry Frontiers. 2017;4(8):1611-14.

Ettaoussi M, Sabaouni A, Rami M, Boutin JA, Delagrange P, Renard P, et al. Design, synthesis and pharmacological evaluation of new series of naphthalenic analogues as melatoninergic (MT1/MT2) and serotoninergic 5-HT2C dual ligands (I). Eur J Med Chem. 2012;49:310-323.

Hirai K, Kita M, Ohta H, Nishikawa H, Fujiwara Y, Ohkawa $\mathrm{S}$, et al. Ramelteon (TAK-375) accelerates reentrainment of circadian rhythm after a phase advance of the light-dark cycle in rats. J Biol Rhythms. 2005;20(1):27-37. 
Kidwai, M, Negi N, Gupta SD. Synthesis and antifertility activity of 1,5-diaryl-3 (3 indolyl) formazans. Chem Pharm Bull. 1994;42(11):2363-2364.

Landagaray E, Ettaoussi M, Duroux R, Boutin JA, Caignard DH, Delagrange $\mathrm{P}$, et al. Melatonergic ligands: Design, synthesis and pharmacological evaluation of novel series of naphthofuranic derivatives. Eur J Med Chem. 2016;109:360-370.

Lemoine P, Zisapel N. Prolonged-release formulation of melatonin (Circadin) for the treatment of insomnia. Expert Opin Pharmacother. 2012;13(6):895-905.

Letra-Vilela R, Sanchez-Sanchez AM, Rocha AM, Martin V, Branco-Santos J, Puente-Moncada N, et al. Distinct roles of $\mathrm{N}$-acetyl and 5-methoxy groups in the antiproliferative and neuroprotective effects of melatonin. Mol Cell Endocrinol. 2016;434:238-49.

Mague JT, Vang S, Berge DG, Wacholtz WF. Isomerism/ tautomerism in hydrazones derived from thiophenaldehydes and 2-hydrazinoquinoline. Acta Cryst. 1997;53:973-979.

Maharaj DS, Maharaj H, Antunes EM, Maree DM, Nyokong T, Glass BD, Daya S. 6-Hydroxymelatonin protects against quinolinic-acidinduced oxidative neurotoxicity in the rat hippocampus. J Pharm Pharmacol. 2005;57(7):877-81.

Mossman T. Rapid colorimetric assay for cellular growth and survival: Application to proliferation and cytotoxicity assays. J Immunol Methods. 1983;65(1-2):55-63.

Niles AL, Moravec RA, Riss TL. Update on in vitro cytotoxicity assays for drug development. Expert Opin Drug Discov. 2008;3(6):655-669.

Puskullu M.O, Shirinzadeh H, Nenni M, Gurer-Orhan H, Suzen S. Synthesis and evaluation of antioxidant activity of new quinoline-2-carbaldehyde hydrazone derivatives: bioisosteric melatonin analogues. J Enzyme Inhib Med Chem. 2015;31(1):121-125.

Reiter RJ, Tan DX, Cabrera J, D'Arpa D, Sainz RM, Mayo JC, et al. The oxidant/antioxidant network: role of melatonin. Biol Signals Recept. 1999;8(1-2):56-63.

Reiter RJ, Tan DX, Osuna C, Gitto E. Actions of melatonin in the reduction of oxidative stress. A review. J Biomed Sci. 2000;7(6):444-458.
Robev S. Rearrangement of aromatic aldehyde arylhydrazones leading to benzimidazole derivatives. Dokl. Bolg. Akad. Nauk. 1981;34(5):663-666.

Robev S. Synthesis of some halogenated aromatic N-arylsubstituted amidines. Dokl Bolg Akad Nauk. 1968;21(11):11813.

Shirinzadeh H, Eren B, Gurer-Orhan H, Suzen S, Ozden S. Novel indole-based analogs of melatonin: synthesis and in vitro antioxidant activity studies. Molecules. 2010;15(4):2187-2202.

Shirinzadeh H, Ince E, Westwell AD, Gurer-Orhan H, Suzen S. Novel indole-based melatonin analogues substituted with triazole, thiadiazole and carbothioamides: studies on their antioxidant, chemopreventive and cytotoxic activities. J Enzyme Inhib Med Chem. 2016;31(6):1312-1321.

Soltani Rad MN, Khalafi-Nezhad A, Behrouz S. Synthesis of some novel hydrazono acyclic nucleoside analogues. Beilstein J Org Chem. 2010;6(49):1-8.

Spadoni G, Diamantini G, Bedini A, Tarzia G, Vacondio F, Silva C, et al. Synthesis, antioxidant activity and structureactivity relationships for a new series of 2-(N-acylaminoethyl) indoles with melatonin-like cytoprotective activity. J Pineal Res. 2006;40(3):259-269.

Suzen S, Tekiner-Gulbas B, Shirinzadeh H, Uslu D, Gurer-Orhan $\mathrm{H}$, Gumustas M, et al. Antioxidant activity of indole-based melatonin analogues in erythrocytes and their voltammetric characterization. J Enzyme Inhib Med Chem. 2013;28(6):11431155 .

Suzen S. Antioxidant activities of synthetic indole derivatives and possible activity mechanisms. Top Heterocycl Chem. 2007;11:145-178.

Suzuki N, Somei M, Seki A, Reiter RJ, Hattori A. Novel bromomelatonin derivatives as potentially effective drugs to treat bone diseases. J Pineal Res. 2008;45(3):229-234.

Valko M, Leibfritz D, Moncol J, Cronin MT, Mazur M, Telser J. Free radicals and antioxidants in normal physiological functions and human disease. Int J Biochem Cell Biol. 2007;39(1):44-84.

Weil H, Ostermeier H. Reduction of naphthalene and naphtholcarboxylic acids. Ber Dtsch Chem Ges B. 1921;54B:3271-9. 
Yamashita T, Sakamoto K, Yamanishi H, Totani N, Yamamoto $\mathrm{J}$. Effect of a free radical scavenger on nitric oxide release in microvessels. Vasc Pharmacol. 2013;58(1-2):134-9.

Y1lmaz AD, Coban T, Suzen S. Synthesis and antioxidant activity evaluations of melatonin-based analogue indolehydrazide/hydrazone derivatives. J Enzyme Inhib Med Chem. 2012;27(3):428-36.
Yonei Y, Hattori A, Tsutsui K, Okawa M, Ishizuka B. Effects of Melatonin: Basics Studies and Clinical Application. Anti-Aging Med. 2010;7(7):85-91.

Received for publication on $26^{\text {th }}$ March 2018 Accepted for publication on $02^{\text {nd }}$ January 2019 